\title{
A Fuzzy Controller for Thermal Comfort and Indoor Air Quality in a Bioclimatic Building*
}

\author{
María D. Bretones ${ }^{1}$, José D. Álvarez ${ }^{1}$, María del Mar Castilla ${ }^{1}$ and Manuel Berenguel ${ }^{1}$
}

\begin{abstract}
Global warming and greenhouse effect are main concerns of actual society. Governments are putting their efforts in reducing $\mathrm{CO}_{2}$ emissions. One way to do this is to save energy in the building sector, which is mainly spent by Heating, Ventilation and Air Conditioning (HVAC) systems. On the other hand, energy saving in buildings cannot put users' welfare at risk. Therefore, a trade-off between energy saving and comfortable environments is desirable. Control systems are suitable for this aim. This work presents a fuzzy controller to maintain both, thermal comfort and indoor air quality, in a laboratory which is part of a bioclimatic building. Several simulation results obtained through a simulator calibrated using real data are showed and commented.
\end{abstract}

\section{INTRODUCTION}

Nowadays, people are more and more concerned with greenhouse effect and global warming. People and governments put their focus in $\mathrm{CO}_{2}$ emissions by the industrial field or the automotive sector among others. However, the building sector is usually forgotten in these discussions which is a mistake mainly due to two reasons: i) people spend most part of the day in dwellings and, ii) the building sector is responsible for $40 \%$ of the total world energy consumption, more than half used by Heating, Ventilation and Air Conditioning (HVAC) systems.

Therefore, energy saving in this field can significantly contribute to reduce the risk derived from global warming. However, people's productivity and health are directly linked with their comfort sensation, and thus, any step taken to reduce the energy spend in buildings must not put users' welfare at risk. Hence, people's comfort must be taken into account and comfortable environments must be ensured from both, thermal and air quality points of view.

In literature, there are several works related to control systems or strategies which are suitable to fulfil these aims, i.e. to save energy and to provide comfortable environments. In the last years, several control strategies have been tested with this purpose, among them it is possible to mention: in [1] an artificial intelligent algorithm is proposed to maintain comfort sensation within some established limits while the energy consumption derived from the use of the HVAC system is reduced; in [2] a Model Predictive Control (MPC) approach is developed to regulate thermal comfort and, simultaneously, to reduce peak load. In addition, some

\footnotetext{
*This work has been funded by the National $\mathrm{R}+\mathrm{D}+\mathrm{i}$ Plan Project DPI2017-85007-R of the Spanish Ministry of Science, Innovation and Universities and ERDF funds.

${ }^{1}$ Centro Mixto CIESOL, ceiA3, Universidad de Almería. Ctra. Sacramento s/n, Almería 04120, Spain; maria_32_behotmail.com; $\{$ jhervas, mcastilla, beren\} @ual.es
}

works takes into consideration the stochastic behaviour of the controlled process, and thus, they present some stochastic control approaches to manage users' comfort and energy consumption [3], [4].

Moreover, it is possible to find some works which use fuzzy control methodologies or rule-based systems applied to comfort control in buildings [5]. For instance, in [6] a fuzzy control system to regulate thermal comfort and optimize the use of the HVAC system is presented. In [7] a fuzzy logic control system to perform an efficient management of the illuminance system is developed. Similarly, in [8] a fuzzy logic based strategy has been used to manage the HVAC systems and humidifying devices in smart buildings in order to reduce energy consumption. In [9] a thermal comfort fuzzy controller which uses the Predicted Mean Vote (PMV) comfort index is presented. In addition, this strategy is compared to a PID controller. The previous works focus on thermal comfort, on visual comfort or energy consumption management but, up to our knowledge, there are not recent works using a fuzzy controller to control both thermal comfort and indoor air quality.

In this work, a fuzzy controller [10] to maintain both, thermal comfort and air quality in a room of a bioclimatic building, is proposed. A couple of rule sets for the fuzzy controller have been designed, one for the summer season and another one for the winter period. Moreover, these sets of rules take into account the temperature difference between outdoor and indoor air in order to save energy. Several tests obtained through simulations with real data gathered from the bioclimatic building, are presented to show the goodness of the proposed control strategy.

The rest of the paper is organized as follows: in Section II the model of the bioclimatic building which has served as a test-bed for the fuzzy controller is described. Besides, the comfort indexes used to estimate thermal comfort and indoor air quality are explained. The proposed control architecture is presented in Section III whereas the results obtained in simulation with this control architecture are commented in Section IV. Finally, in Section V the main conclusions are summarized.

\section{SCOPE OF THE RESEARCH}

As it was mentioned before, this paper presents the results provided by a rule-based controller developed to maintain thermal comfort and indoor air quality environmental conditions inside a bioclimatic building. More in detail, this control system has been tested through simulations into a representative room of the CIESOL building. 
The CIESOL building (www.ciesol.es), see Figure 1(a) is a research centre placed in the South-East of Spain. It was built following a bioclimatic approach. Hence, it counts with some passive and active strategies which try to harness solar energy in order to reduce energy consumption. Besides, this building is completely monitored through a wide network of sensors that include, among others, air temperature, relative humidity and $\mathrm{CO}_{2}$ concentration measurements. The room selected to test the control strategy presented in this paper is used as an office and it is located in the first floor of the building between two rooms with analogous characteristics, see Figure 1(b). As main actuators, it has an automated window situated in the wall which faces North and a fancoil unit for heating and cooling.

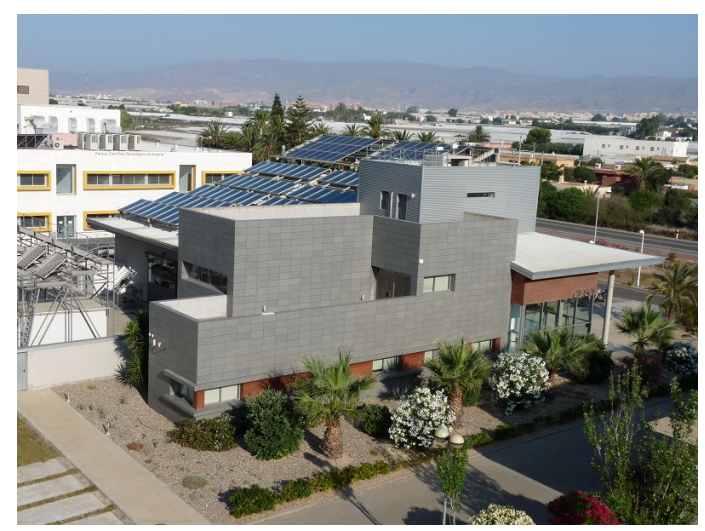

(a) Overview of the building

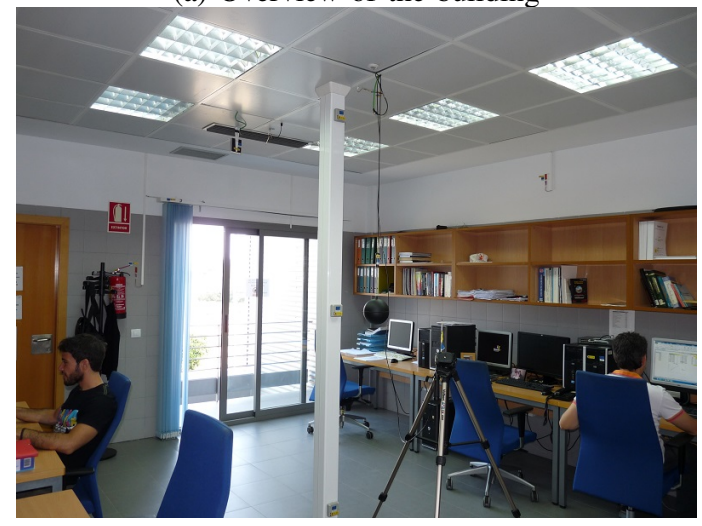

(b) Room selected to test the rule-based controller

Fig. 1. CIESOL building

This section makes emphasis in the definition of thermal comfort and indoor air quality, and how they can be quantified. In addition, a brief description of the first principles model of the selected room which has been used as simulator has been included.

\section{A. Thermal comfort}

Thermal comfort can be described as "that mental condition which expresses satisfaction with the surrounding thermal environment" [11]. Nonetheless, not everyone perceives this sensation in a similar manner since it is strongly related to psychological and physiological processes. Despite that, it is possible to determine the appropriate thermal environmental conditions for a specific percentage of occupants from a series of statistical data obtained through surveys. These conditions should consider parameters as the operating temperature, relative humidity limits, air drafts, local thermal discomfort, etc. [12].

One of the most widespread indexes in literature is the the Predicted Mean Vote (PMV) proposed by Fanger in the seventies [11]. This index expresses the mean thermal satisfaction of a group of people according to a sevenpoints scale which varies from -3 (cold sensation) to +3 (hot sensation). The international standard ISO 7730 [13] recommends for a neutral thermal comfort sensation a PMV index value equals to 0 with a tolerande of \pm 0.5 . More in detail, the PMV index is estimated as a function of four environmental variables (air temperature, relative humidity, mean radiant temperature and air velocity) and two peopledependent variables (metabolic rate and clothing insulation). A detailed explanation of the methodology followed to obtain this index has not been included in this paper due to lack of space, but it can be found in [14].

\section{B. Indoor air quality}

A poor indoor air quality inside buildings can lead to the appearance of the Sick Building Syndrome (SBS) [15]. SBS causes health problems and acute discomfort related to the time spent inside the building such as headaches, dry coughs, fatigue, nose, throat or eye irritation, etc. It can be originated by both chemical contaminants (volatile organic pollutants, carbon dioxide $\left(\mathrm{CO}_{2}\right)$, carbon monoxide, tobacco smoke, etc.) or biological ones (infectious agents, antigens and toxins). The source of these contaminants is very diverse: combustion processes, building materials, human beings and animals, etc.

Therefore, the availability of a good indoor air quality is a cornerstone which can considerably improve users' comfort. According to the international standard CEN EN13779 [16], indoor air quality can be classified into four different categories: i) High Indoor Air Quality (IDA-1); ii) Average Indoor Air Quality (IDA-2); iii) Moderate Indoor Air Quality (IDA-3); and iv) Low Indoor Air Quality (IDA4).

Furthermore, as people spend most of their time inside buildings, in this work the $\mathrm{CO}_{2}$ concentration has been selected as indicator of indoor air quality since it is the main effluent resulting from human respiration. More in detail, it is possible to find in literature a direct relationship between the categories of indoor air quality and $\mathrm{CO}_{2}$ concentration, see Table I.

\section{Room simulator: A model based on first principles}

The controller presented in this paper has been evaluated by means of simulation tests. To do that, a simulator based on a first principles model has been used. More specifically, the room has been defined as a system made up of walls, a window, a fancoil unit, etc. Then, the relationships existing among these elements and the main involved environmental 
TABLE I

RECOMMENDED INDOOR $\mathrm{CO}_{2}$ LEVELS IN $[p p m]$ BASED OF INDOOR AIR QUALITY CATEGORIES [16]

\begin{tabular}{|c||c||c|}
\hline Category & Typical range & Default values \\
\hline IDA-1 & $\leq 400$ & 350 \\
\hline IDA-2 & $400-600$ & 500 \\
\hline IDA-3 & $600-1000$ & 800 \\
\hline IDA-4 & $>1000$ & 1200 \\
\hline
\end{tabular}

variables, that is, indoor air temperature and $\mathrm{CO}_{2}$ concentration have been modelled by means of mass transfer and heat transfer laws. Therefore, the room simulator is composed of a set of differential equations as it is shown in Eq.(1):

$$
\frac{d X}{d t}=f(X ; U ; D ; V ; C ; t) \text { with } X\left(t_{i}\right)=X_{i}
$$

In the previous equation, $X, U, D, V$, and $C$ represent vectors of state variables, control input variables, disturbances, system variables and system constants, respectively. $X_{i}$ represents the initial state at time $t_{i}$, and finally, $f$ is a nonlinear function defined by heat and mass transfer balances.

More concretely, the indoor air temperature, $T_{\text {air }}$, first principles model, see Eq. (2), depends on the heat exchanged through the different elements and processes involved in the room: i) the walls of the room $\left(Q_{\text {conv }}\right)$; ii) the window $\left(Q_{\text {glass }}\right)$; iii) the fancoil unit $\left(Q_{H V A C}\right)$; iv) natural ventilation $\left.\left(Q_{n v n t}\right) ; \mathrm{v}\right)$ infiltration $\left(Q_{\text {inf }}\right)$ and vi) internal gains $\left(Q_{i \text { Gain }}\right)$, that is, the influence of people, artificial lighting and other electrical appliances.

$$
\begin{aligned}
m_{\text {air }} \cdot C_{p_{\text {air }}} \cdot \frac{d T_{\text {air }}}{d t} & =Q_{\text {conv }}+Q_{\text {glass }}+Q_{H V A C} \\
& +Q_{n v n t}+Q_{\text {inf }}+Q_{i \text { Gain }}
\end{aligned}
$$

where $m_{\text {air }}$ is the mass of air in the room and $C_{p_{\text {air }}}$ is the specific heat capacity of air. Besides that, to model $\mathrm{CO}_{2}$ concentration, it has been necessary to consider the influence of people $\left(N_{p}\right)$ and their physical activity since it will affect their $\mathrm{CO}_{2}$ generation rate $\left(\mathrm{G}_{\mathrm{CO}_{2}}\right)$. Moreover, the air flow through intentional or non-intentional apertures have been considered, that is, through the window $\left(q_{n v n t}\right)$, the fancoil unit $\left(q_{H V A C}\right)$ and by means of infiltrations $\left(q_{\text {inf }}\right)$. The resulting equation can be observed in Eq. (3).

$$
\begin{aligned}
V_{a} \cdot \frac{d C O_{2_{i n}}}{d t} & =N_{p} \cdot G_{C O_{2}}+q_{H V A C} \cdot C O_{2_{\text {doubleceil }}} \\
& +\left(q_{n v n t}+q_{\text {inf }}\right) \cdot C O_{2_{\text {out }}} \\
& -\left(q_{\text {nvnt }}+q_{\text {inf }}+q_{H V A C}\right) \cdot C O_{2_{\text {in }}}
\end{aligned}
$$

where $V_{a}$ is the volume of air in the room, $\mathrm{CO}_{2_{\text {in }}}$ and $\mathrm{CO}_{2_{\text {out }}}$ are the inside and outside carbon dioxide concentrations, respectively, and $\mathrm{CO}_{2 \text { doubleceil }}$ that of the double ceil. As can be observed in Eqs. (2) and (3), both models are coupled by means of several variables such as the number of people, the use of the HVAC system or natural ventilation through the window. A detailed explanation of these models and the room simulator used to test the controller presented in this paper can be found in [14], [17].

\section{CONTROL ARCHITECTURE}

It was in 1965 when Lofti A. Zadeh introduced the concept of fuzzy sets [10], where unlike in classical logic, a degree to which a certain object belongs to a set was attributed, instead of only two states, as true or false [18], [19].

Fuzzy Logic is a technique that imitates human behaviour for decision making through the definition of fuzzy sets and intuitive rules. Thus, this technique allows using ambiguous and inaccurate information, such as the one used in natural language, and, by this way, a computer can deal with this information. Moreover, the system is able to make the appropriate decisions from inaccurate data, just like an expert operator would. One of the advantages of this technique is that it is easy to implement since it does not rely on a mathematical basis and the rules are created from prior experience of the operator, who can quickly update its parameters when the system requires it.

Controllers based on Fuzzy Logic are able to emulate human thinking thanks to its knowledge base. This base contains the set of rules which the system is able to manage, that is, to provide the appropriate inputs to the actuators to reach a desirable set-point.

These controllers are mainly used when the problem to be controlled and its mathematical model are complex or nonlinear. Thus, these controllers can be designed from experience, usually providing very effective and efficient results when compared to other types of controllers. For this reason, this kind of controllers is suitable to maintain users' comfort inside buildings as has been shown in [20], [21]. In this work, thermal comfort and indoor air quality are controlled simultaneously and, moreover, some rules have been introduced in order to save energy.

\section{A. Input and output variables}

The input variables are: i) the integral of the PMV index which is helpful to estimate thermal comfort, ii) the indoor $\mathrm{CO}_{2}$ concentration as a measurement of indoor air quality and, iii) the difference between the outdoor and indoor air temperatures, $\left(\mathrm{T}_{a m b}-\mathrm{T}_{i n}\right)$, in order to allow also the use natural ventilation by opening the window to regulate the indoor temperature and, thus, reduce the electrical consumption associated to the HVAC.

It is important to highlight that, the integral of the PMV index has been used instead of the value of the PMV since the fuzzy controller has been designed to maintain the PMV value as close to zero as possible, i.e., optimal thermal comfort. Several simulation tests using the PMV value as an input to the fuzzy controller allowed us to maintain the PMV value into the comfort zone, i.e, between -0.5 and +0.5 , but not close to 0 . Thus, using the integral of the PMV steadystate errors are removed.

On the other hand, the output variables must be able to regulate and maintain both, thermal comfort and indoor air quality. For these reasons, one of the output variables will be 
the air velocity through the fancoil (Fancoil), which is able to cool or heat the room and the other one will be the window opening (WO), through which it is possible to ventilate the room and to get a less foul air, and, therefore, a better indoor air quality.

The input and output variables, as well as, their ranges are listed in Table II.

TABLE II

FUZZY CONTROL VARIABLES

\begin{tabular}{|l|l|l|l|}
\hline Variable type & Name & Range & Unit \\
\hline \multirow{3}{*}{ Input variable } & $\int \mathrm{PMV}$ & $-3+3$ & - \\
\cline { 2 - 4 } & $\mathrm{CO}_{2}$ & $0-1500$ & $\mathrm{ppm}$ \\
\cline { 2 - 4 } & $\mathrm{T}_{a m b}-\mathrm{T}_{\text {in }}$ & $-22+22$ & ${ }^{\circ} \mathrm{C}$ \\
\hline \multirow{2}{*}{ Output variable } & Fancoil & $0-3$ & $\mathrm{~m} / \mathrm{s}$ \\
\cline { 2 - 4 } & $\mathrm{WO}$ & $0-100$ & $\%$ \\
\hline
\end{tabular}

\section{B. Linguistic variables}

Quantification levels must cover the whole range of controller input and output variables. This is achieved with the linguistic variables assigned to each input and output variable. In Tables III and IV, the linguistic variables and its abbreviations for each controller input and output variable are shown, respectively.

TABLE III

LINGUISTIC VARIABLES FOR THE CONTROLLER INPUT VARIABLES

\begin{tabular}{|l|l|l|}
\hline Input variable & \multicolumn{2}{l|}{ Linguistic variable } \\
\hline \multirow{5}{*}{$\int$ PMV } & GN & Big Negative \\
\cline { 2 - 3 } & MN & Medium Negative \\
\cline { 2 - 3 } & PN & Small Negative \\
\cline { 2 - 3 } & OK & Satisfactory \\
\cline { 2 - 3 } & PP & Small Positive \\
\cline { 2 - 3 } & MP & Medium Positive \\
\cline { 2 - 3 } $\mathrm{CO}_{2}$ & GP & Big Positive \\
\hline \multirow{5}{*}{$\mathrm{T}_{a m b}-\mathrm{T}_{i n}$} & A & High \\
\cline { 2 - 3 } & M & Medium \\
\cline { 2 - 3 } & MO & Moderate \\
\cline { 2 - 3 } & B & Low \\
\hline & GN & Big Negative \\
\cline { 2 - 3 } & PN & Small Negative \\
\cline { 2 - 3 } & OK & Satisfactory \\
\cline { 2 - 3 } & PP & Small Positive \\
\cline { 2 - 3 } & GP & Big Positive \\
\hline
\end{tabular}

\section{Membership functions}

The membership functions used in this work have been triangular and trapezoidal ones, since, due to their simplicity, mathematical calculations are simplified while the accuracy is maintained. They are also often used when the system has considerable changes in its behaviour in short periods of time [22]. On the other hand, with respect to the trapezoidal function, the uncertainty is reduced in the area for which the values of $x$ have a membership degree of 1 (upper base of the trapezoid), reducing the actuators operating time [21].

In Fig. 2 the membership functions for the input variables are showed. More in detail, the upper figure displays the membership function for the integral of the PMV index,
TABLE IV

LINGUISTIC VARIABLES FOR THE CONTROLLER OUTPUT VARIABLES

\begin{tabular}{|l|l|l|}
\hline Output variable & \multicolumn{2}{|l|}{ Linguistic variable } \\
\hline \multirow{4}{*}{ Fancoil } & OFF & Shutdown \\
\cline { 2 - 3 } & B & Low \\
\cline { 2 - 3 } & M & Medium \\
\cline { 2 - 3 } & A & High \\
\cline { 2 - 3 } & ON & Maximum \\
\hline \multirow{5}{*}{ WO } & OFF & Closed \\
\cline { 2 - 3 } & CC & Almost closed \\
\cline { 2 - 3 } & SC & Semi-closed \\
\cline { 2 - 3 } & M & Medium \\
\cline { 2 - 3 } & CA & Almost open \\
\cline { 2 - 3 } & ON & Open \\
\hline
\end{tabular}

in the middle figure the membership function for the $\mathrm{CO}_{2}$ concentration is showed. Finally, the bottom figure depicts the membership function for the temperature difference.

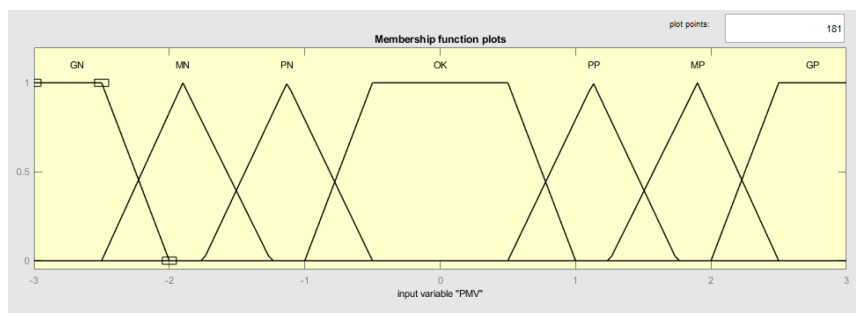

(a) Membership function for the integral of the PMV

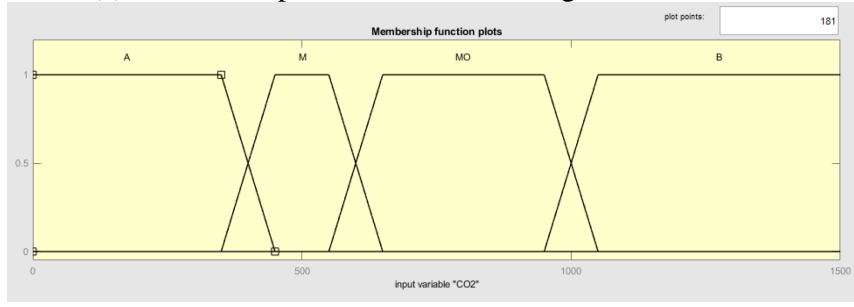

(b) Membership function for the $\mathrm{CO}_{2}$ concentration

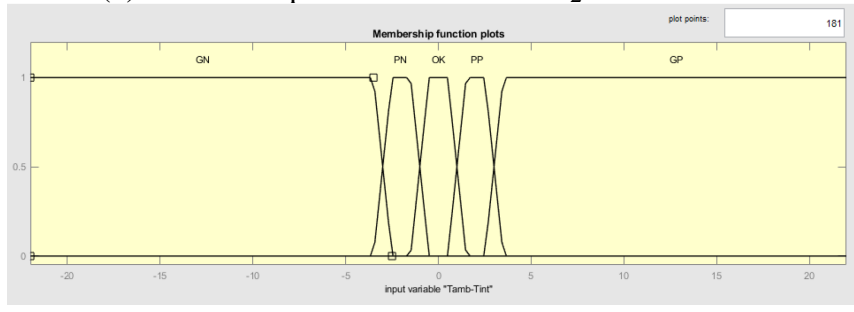

(c) Membership function for the temperature difference

Fig. 2. Membership functions for the input variables

It is important to note that, triangular functions have been used to represent the integral of the PMV linguistic variables, with the exception of the area where comfort is acceptable (a PMV index within the range from -0.5 to 0.5 ), and where the feeling of comfort is considered to be totally inadequate (a PMV index in the range from -2.5 and 2.5), because it is too cold or warm. In these cases, trapezoidal functions are more suitable.

On the other hand, the representation of the $\mathrm{CO}_{2}$ concentration linguistic variables has been carried out through trapezoidal membership functions, since each one completely 
covers the four categories of IDA (A: $\leq 400$, M: 400 600, MO: $600-1000$ and $\mathrm{B}>1000(\mathrm{ppm}))$, where the cutoff point of these functions represents the limits of the categories, being 400, 600 and 1000 .

Finally, for the difference temperature, i.e. $\mathrm{T}_{a m b}-\mathrm{T}_{i n}$, trapezoidal membership functions have been used for the similar reasons that the ones explained for the other input variables.

Regarding the output variables, in Fig. 3 the membership functions for the fancoil and the window are depicted in the upper and bottom picture, respectively.

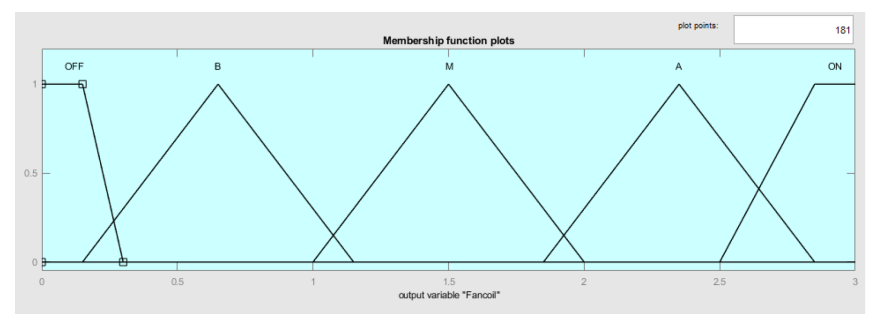

(a) Membership function for the fancoil

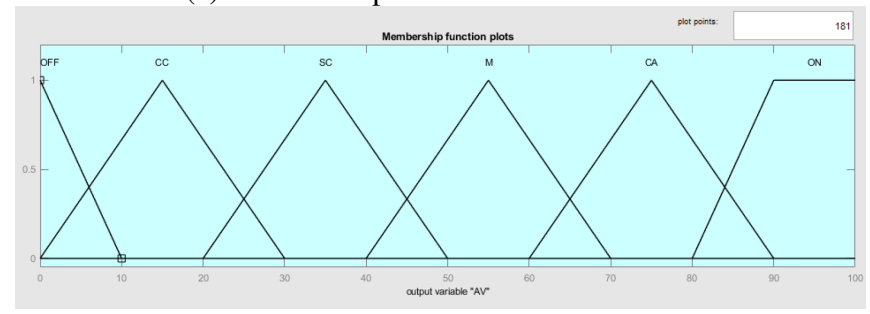

(b) Membership function for the window

Fig. 3. Membership functions for the outputs variables

More specifically, for the fancoil triangular and trapezoidal functions are used. The trapezoidal functions are used at the range limits, since there is a small dead zone where the fancoil is turned on (about $0.15 \mathrm{~m} / \mathrm{s}$ ) and, in addition, it is considered that from $2.85 \mathrm{~m} / \mathrm{s}$ it works at its maximum capacity. On the other hand, the window membership function is represented with triangular functions, with the exception of the linguistic variable ON. This is because it is considered that from $90 \%$ of the window opening it is fully open.

\section{Set of rules}

The set of fuzzy rules is one of the fundamental parts of the system since if the rules are poorly designed the control objective will not be achieved. Thanks to these rules, it is possible to emulate the operator's behaviour. Its construction is done from experience or through trial and error and simulations. In general, it is easy to obtain intuitive rules. Mamdani type fuzzy rules are used in this work. These rules relate all possible combinations of input variables. Therefore, as the developed fuzzy control system has three input variables, the integral of the PMV, the $\mathrm{CO}_{2}$ concentration and $\mathrm{T}_{a m b^{-}}$ $\mathrm{T}_{\text {in }}$ with 7, 4 and 5 linguistic variables respectively, it results in a set of 140 rules.

It is intended to study two different scenarios, one for the summer season and other for the winter period. Therefore, two sets of fuzzy rules have been generated for each scenario. These sets of fuzzy rules have been created from intuition and, later, they have been improved by doing several simulation tests. However, due to the lack of space, the list of fuzzy rules cannot be included in this document. Therefore, a couple of rules from the summer season scenario have been included as an example:

- IF $\left(P M V=G N\right.$ and $C O_{2}=A$ and $\left.\left(T_{a m b}-T_{i n}\right)=P P\right)$ THEN (Fancoil $=O F F$ and $W O=P P$ )

- IF $\left(P M V=G N\right.$ and $C O_{2}=A$ and $\left.\left(T_{a m b}-T_{i n}\right)=G P\right)$ THEN $($ Fancoil $=O F F$ and $W O=S C)$

Moreover, although the main purpose of the control system presented in this paper is to maintain users' comfort, it is worthy to mention that the definition of the sets of fuzzy rules has been performed considering energy saving criteria. Specifically, the input variable $\left(T_{a m b}-T_{i n}\right)$ has been used in order to decrease energy consumption. This input variable allows the controller to decide what actuator is better to use considering their physical limitations and assuming that the energy consumption derived from the use of the fancoil is greater than the one derived from opening the window.

\section{RESULTS AND DISCUSSION}

The fuzzy controller described in Section III has been implemented using the MATLAB Fuzzy Logic Toolbox. In this section, the results obtained with this controller will be commented.

\section{A. Summer season}

For the summer season, several simulations have been done with the aim to test the fuzzy controller in different scenarios. Again, due to the lack of space is not possible to show all of them in the document but the most representative ones. Specifically, two scenarios with different boundary conditions have been tested. Besides, for each scenario a comparison between the performance of the fuzzy based controller (controlled case) and without any controller (uncontrolled case in which both control signals, the fancoil velocity and the window opening, are equal to zero during the whole simulation) is done. It is necessary to mention that the manipulated variables, that is, $\mathrm{PMV}$ and $\mathrm{CO}_{2}$ concentration are controlled jointly.

The first scenario for the summer season consists of four days of real data saved from CIESOL building, used to test in simulation the fuzzy controller. Specifically, these data come from June. The simulation results are depicted in Fig. 4 where the controlled variables are showed and in Fig. 5 where the control variables are depicted. It is important to note that, in the top picture in Fig. 4, the PMV index starts at a value close to 0.4 and, when it is controlled, it decreases until it oscillates around 0 and remains in the optimal comfort zone. On the contrary, when the PMV index is not controlled, it increases until being outside the comfort zone and inside the hot zone, reaching values of 0.8 and 0.9 when people are inside the room, since people provide heat to the ambient. On the other hand, in the bottom picture the concentration of indoor $\mathrm{CO}_{2}$ in the room, both controlled and uncontrolled, is showed. It is possible to note that, under the control system the $\mathrm{CO}_{2}$ concentration remains in the limit of the high indoor 
air quality category (IDA-1), being between 390 and 450 $\mathrm{ppm}$. However, when the $\mathrm{CO}_{2}$ concentration is not controlled, it reaches values closed to $800 \mathrm{ppm}$ due to the entry and exit of people, who are the main source of $\mathrm{CO}_{2}$ throughout the day, reaching a moderate indoor air quality.

Regarding the control variables showed in Fig. 5 the fancoil is not turned on all the time but when is necessary to drive the PMV close to 0 . On the other hand, the window is opened when the fancoil is turned off and the PMV can be maintained through the air that comes into the room since its temperature is less than the indoor air one. It is important to note that, through the fancoil is possible to maintain the $\mathrm{CO}_{2}$ concentration in a desirable level too since when the fancoil is turned on the air inside the room is refreshed.

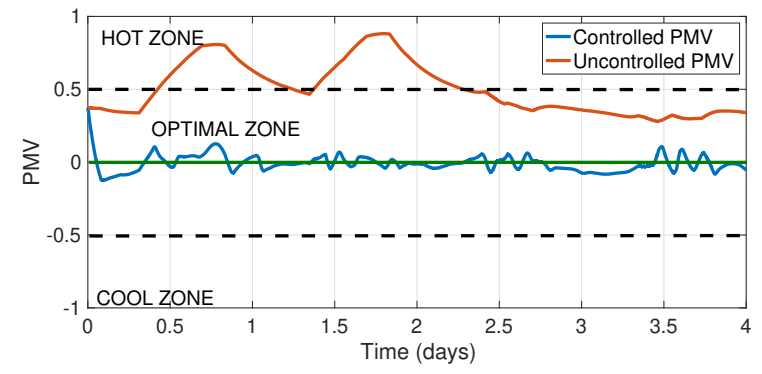

(a) PMV index

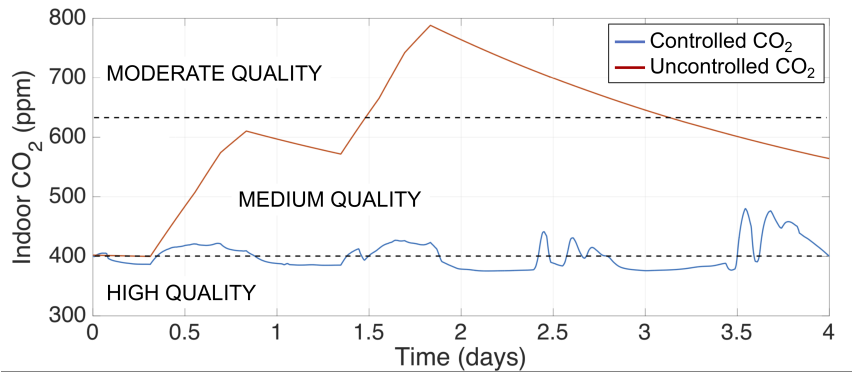

(b) $\mathrm{CO}_{2}$ concentration

Fig. 4. Controlled variables for the summer season. Base case scenario

In the second scenario, the radiant temperatures are increased a couple of Celsius degrees with the aim to test the controller in a more demanding scenario. The results of the PMV index and the control variables are depicted in Fig. 6, while the results of the $\mathrm{CO}_{2}$ concentration are not showed because they are almost the same than in Fig. 4. In this scenario the fancoil is turned on more time than in the base case scenario and the window is opened more times in order to save energy when possible. In addition, the controlled PMV oscillates farther from the 0 value, reaching the upper limit of the comfort zone during the first day. Despite this, the controller is able to keep it into the comfort zone. However, uncontrolled PMV shots up until it reaches a 1.5 value at the end of the second day.

\section{B. Winter season}

For the winter season, two scenarios which make use of the same boundary conditions have been tested. The main difference between both scenarios is that they use

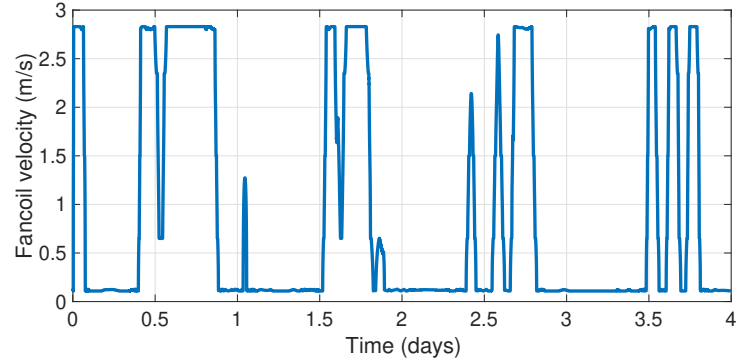

(a) Fancoil velocity

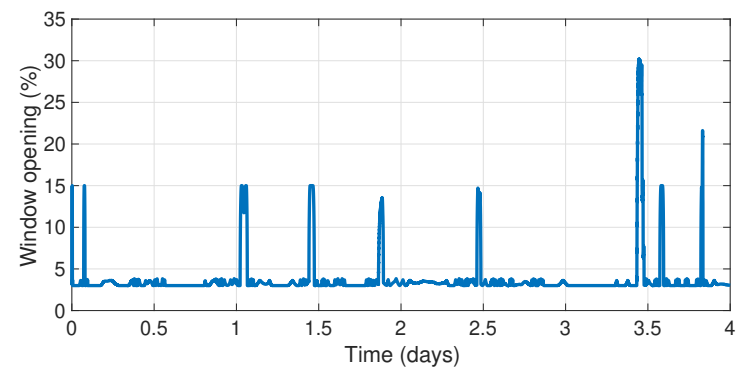

(b) Window opening

Fig. 5. Control variables for the summer season. Base case scenario

different settings for the fuzzy controller. Besides, as for the summer season, for each scenario a comparison between the performance of the fuzzy based controller (controlled case) and without any controller (uncontrolled case) is done. Specifically, for the first winter scenario, other four days of real data from CIESOL buildings are used. However, these data come from the December month.

The simulation results of the fuzzy controller, which are obtained through the set of rules for winter period and with these boundary conditions, are depicted in Fig. 7 and in Fig. 8 where the controlled variables and the control variables are depicted, respectively.

The PMV starts at a value close to -0.2 , and when it is controlled it increases until it oscillates around 0 , staying in the optimal comfort zone. Several fluctuations around the 0 value are due to the repeated turn on and off of the fancoil, since the environmental conditions are highly unfavourable. For this reason, most part of the simulation the fancoil is turned on, Fig. 8(a), and the window is closed since the outdoor temperature is too cold to use it for maintaining the thermal comfort. If the PMV is not controlled, it decreases until standing in the cool zone, especially the last two days, reaching values close to -0.8 which are outside the comfort zone. The areas where the PMV is less negative, coincide with those of greater occupation since people produce heat to the environment with their activity. Figure 7(b) shows the $\mathrm{CO}_{2}$ concentration inside the room, both controlled and uncontrolled. It is noted that, with the control system, it remains in the upper limit of the high indoor air quality category (IDA-1), being between 380 and $410 \mathrm{ppm}$, but when the indoor $\mathrm{CO}_{2}$ concentration is not controlled, it is not kept constant, undergoing increases when there is any person inside the room (first, second and fourth day). 


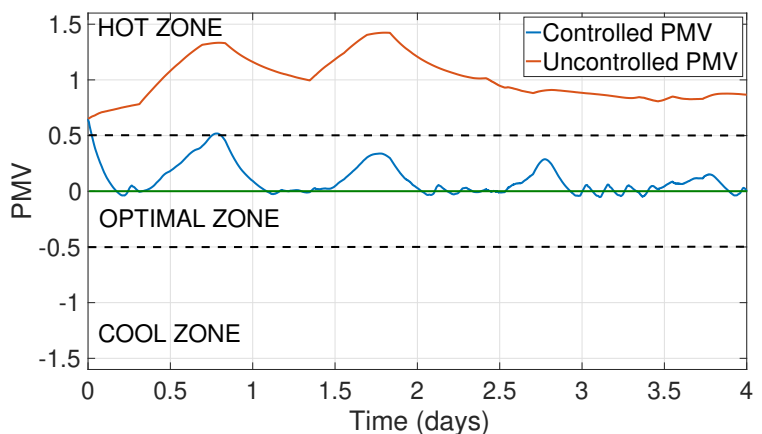

(a) PMV index

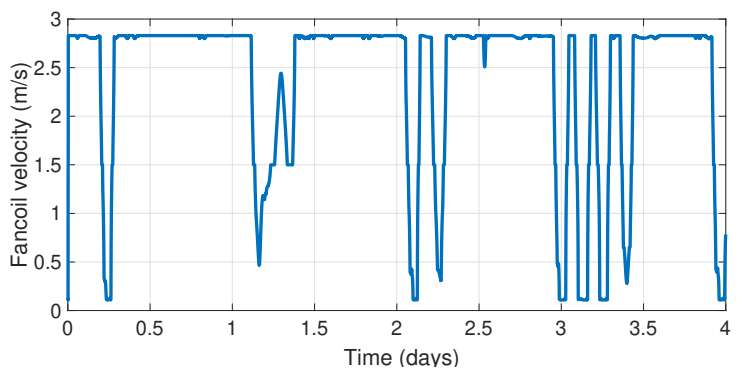

(b) Fancoil velocity

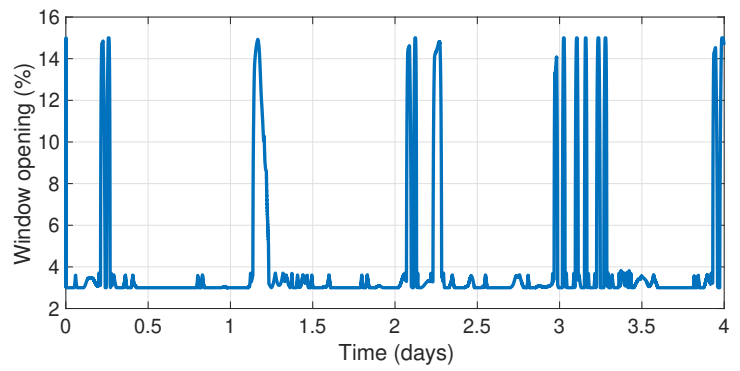

(c) Window opening

Fig. 6. PMV index and control variables for the summer season. Demanding thermal comfort scenario

Among the other simulations performed for the winter period, in Fig. 9 it is possible to see the results obtained with a new setting for the fuzzy controller. In this scenario, the integral of the PMV has been weighted with a factor of $1 / 10$. By this way, it is intended to achieve a less aggressive control. As a consequence of this new configuration, a reduction in the fancoil velocity is observed, see Fig. 9(b). This control signal is less aggressive than the base case, as expected, thus achieving greater energy savings. On the other hand, the window opening is almost non-existent as in the base case, see Fig. 9(c). With this new configuration, the PMV index is still close to 0 most part of the simulation but with fewer fluctuations than in the base case. Thus, greater energy saving is obtained without compromising thermal comfort.

\section{CONCLUSIONS}

The main objective of this work has been the development of a control system to maintain thermal comfort and indoor air quality within their optimal ranges in a room of the CIESOL bioclimatic building, taking into consideration some

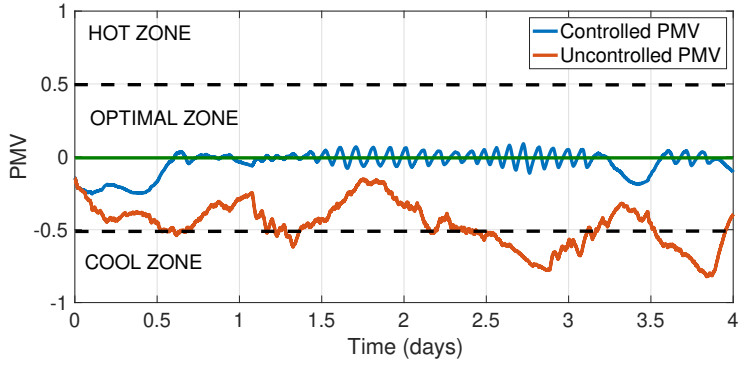

(a) PMV index

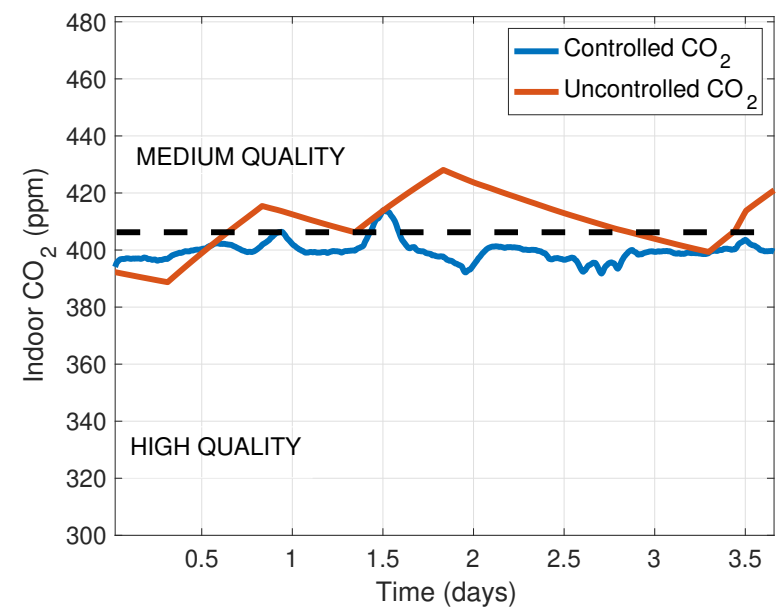

(b) $\mathrm{CO}_{2}$ concentration

Fig. 7. Controlled variables for the winter season. Base case scenario

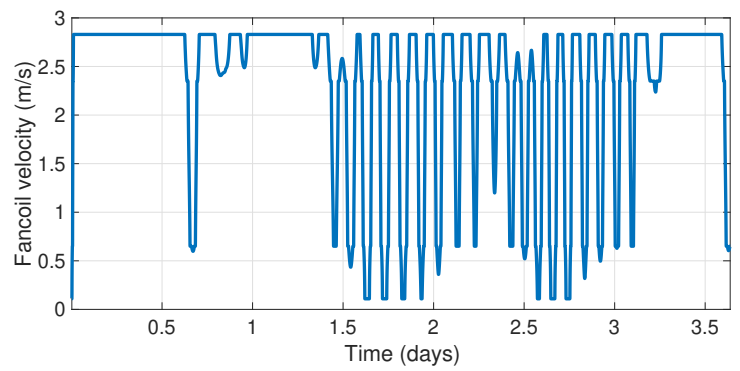

(a) Fancoil velocity

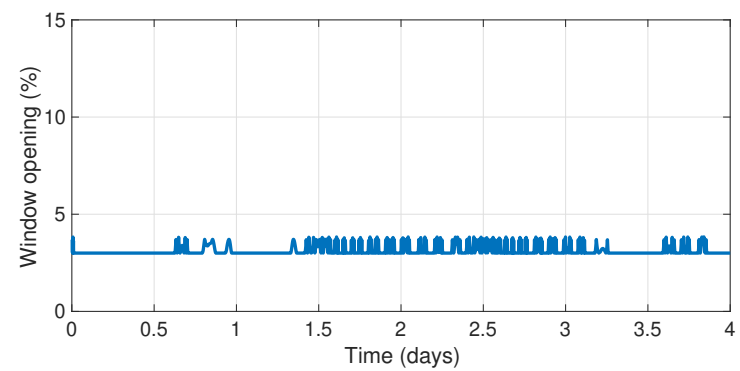

(b) Window opening

Fig. 8. Control variables for the winter season. Base case scenario

energy saving constraints. This objective has been addressed through a fuzzy controller, which allows us to emulate human reasoning with the definition of a set of intuitive rules, so that, the control system makes the appropriate decisions. A 


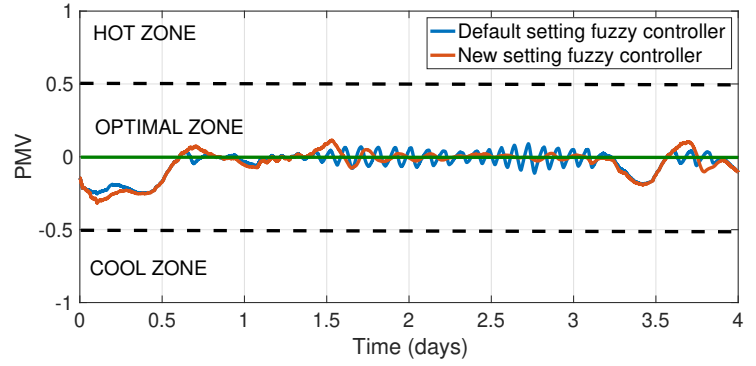

(a) PMV index

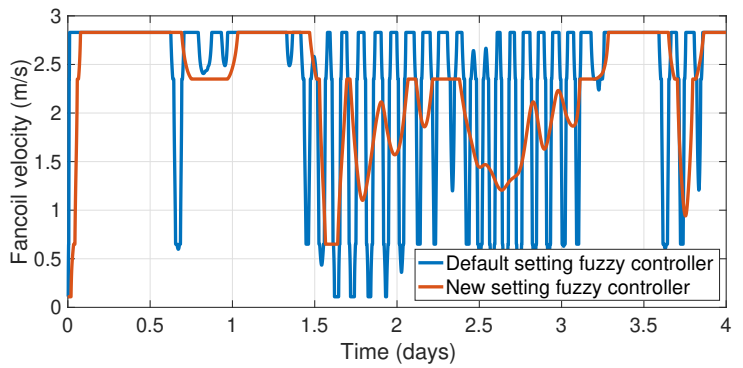

(b) Fancoil velocity

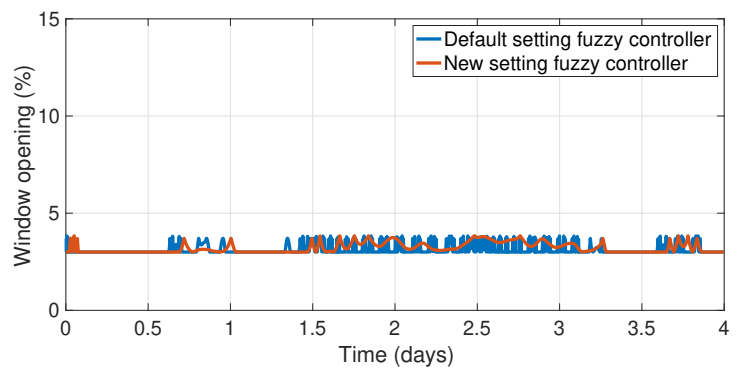

(c) Window opening

Fig. 9. PMV index and control variables for the winter season. New setting for the fuzzy controller scenario

couple of sets of rules have been designed for summer and winter seasons.

After that, several scenarios have been considered changing the environmental variables. In all of them, the results were satisfactory since the controller was able to maintain the PMV index, which is used to estimate thermal comfort, within its optimal zone and, at the same time, high indoor air quality was provided. Moreover, these results validate the setting of the fuzzy controller and the choice of the input and output variables as well as their membership functions among others.

Future works deal with the implementation of the fuzzy controller in the Supervisory Control And Data Acquisition (SCADA) system of the CIESOL building in order to obtain results in real conditions.

\section{REFERENCES}

[1] Valladares, W., Galindo, M., Gutierrez, J., Wu, W. C., Liao, K. K., Liao, J. C., and Wang, C. C. (2019). Energy optimization associated with thermal comfort and indoor air control via a deep reinforcement learning algorithm. Building and Environment, 155, 105-117.

[2] Biyik, E., and Kahraman, A. (2019). A predictive control strategy for optimal management of peak load, thermal comfort, energy storage and renewables in multi-zone buildings. Journal of Building Engineering, 100826.
[3] Latif, M., and Nasir, A. (2019). Decentralized stochastic control for building energy and comfort management. Journal of Building Engineering, 24, 100739.

[4] Shaikh, P. H., Nor, N. B. M., Nallagownden, P., and Elamvazuthi, I. (2014). Stochastic optimized intelligent controller for smart energy efficient buildings. Sustainable Cities and Society, 13, 41-45.

[5] Merabti, S., Draoui, B., and Bounaama, F. (2016, November). A review of control systems for energy and comfort management in buildings. In 2016 8th International Conference on Modelling, Identification and Control (ICMIC) (pp. 478-486). IEEE.

[6] Hussain, S., Gabbar, H. A., Bondarenko, D., Musharavati, F., and Pokharel, S. (2014). Comfort-based fuzzy control optimization for energy conservation in HVAC systems. Control Engineering Practice, $32,172-182$.

[7] Khalid, R., Abid, S., Zafar, A., Yasmeen, A., Ali Khan, Z., Qasim, U. and Javaid, N. (2017). Fuzzy energy management controller for smart homes. In International Conference on Innovative Mobile and Internet Services in Ubiquitous Computing. Springer, Cham, 2017. p. 200-207.

[8] Omarov, B., Altayeva, A., Suleimenov, Z., Im Cho, Y., and Omarov, B. (2017). Design of fuzzy logic based controller for energy efficient operation in smart buildings. In 2017 First IEEE International Conference on Robotic Computing (IRC), 346-351. IEEE.

[9] Ciabattoni, L., Cimini, G., Ferracuti, F., Ippoliti, G., and Longhi, S. (2016). Indoor Thermal Comfort Control Based on Fuzzy Logic. In Advances in Chaos Theory and Intelligent Control, 829-850, Springer, Cham.

[10] Zadeh, L. A. (1965). Fuzzy sets. Information and Control, 8(3), 338-353.

[11] Fanger, P. O. (1970). Thermal comfort. Analysis and applications in environmental engineering. Thermal comfort. Analysis and applications in environmental engineering. McGraw Hill, New York.

[12] Standard, A. S. H. R. A. E. (2017). 55, Thermal environmental conditions for human occupancy. American Society of Heating, Refrigerating and Air conditioning Engineers.

[13] International Organization for Standardization. (2006). ISO 7730: Ergonomics of the thermal environment - Analytical determination and interpretation of thermal comfort using calculation of the PMV and PPD indices and local thermal comfort criteria. ISO.

[14] Castilla, M., Álvarez, J. D., Rodríguez, F. and Berenguel, M (2014). Comfort Control in Buildings. Advances in Industrial Control. Springer.

[15] Redlich, C. A., Sparer, J.and Cullen, M. R. (1997). Sick-building syndrome. The Lancet, 349(9057), 1013-1016.

[16] CEN, E. (2005). Ventilation for non-Residential Buildings - Performance Requirements for Ventilation and Room-Conditioning Systems. In CEN, EN 13779. European Committee for Standardization Brussels.

[17] Castilla, M., Bonilla, J., Álvarez, J. D. and Rodríguez, F. (2016). A room simulation tool for thermal comfort control in a bioclimatic building: A real example of use with an optimal controller. Optimal Control Applications and Methods, 37(3), 479-495.

[18] Zadeh, L. A. (1973). Quantitative fuzzy semantics. Information Sciences, 3, 159-176.

[19] Zadeh, L. A. (1988). Fuzzy logic. Computer, 21(4), 83-93.

[20] Dounis, A. I., Bruant, M., Santamouris, M., Guarracino, G. and Michel, P. (1996). Comparison of conventional and fuzzy control of indoor air quality in buildings. Journal of Intelligent \& Fuzzy Systems, 4(2), 131-140.

[21] Dounis, A. I. and Manolakis, D. E. (2001). Design of a fuzzy system for living space thermal-comfort regulation. Applied Energy, 69(2), 119-144.

[22] Bai, Y. and Wang, D. (2006). Fundamentals of fuzzy logic control-fuzzy sets, fuzzy rules and defuzzifications. In Advanced Fuzzy Logic Technologies in Industrial Applications (pp. 17-36). Springer, London. 